\title{
Linking $\gamma$-ray Observations with Models of eta Carinae
}

\author{
O. Reimer ${ }^{* a}$, R. Kissmann ${ }^{a}$, A. Reimer ${ }^{b}$, and K. Reitberger ${ }^{a}$ \\ ${ }^{a}$ Institute for Astro- and Particle Physics, Innsbruck University, Austria \\ ${ }^{a}$ Institute for Theoretical Physics, Innsbruck University, Austria \\ E-mail: \\ olaf.reimer@uibk.ac.at,ralf.kissmann@uibk.ac.at,anita.reimer@uibk.ac.at, \\ klaus.reitberger@uibk.ac.at
}

Eta Carinae, the so-far only colliding wind binary detected at high-energy gamma-rays, has been observed over its first complete orbit since launch of Fermi Gamma-ray Space Telescope. Different emission scenarios were proposed to explain the temporal and spectro-temporal features, by either postulating strong dominance of hadronic particle populations, a single emitting particle population in combination with significant absorption by $\gamma-\gamma$ pair production, or mixed emission scenarios. The interplay between different particle populations as function of energy, orbital phase, inclination etc. is best studied by hydrodynamical simulations in conjunction with particle transport models. We demonstrate that transitions between hadron-dominated to leptondominated high-energy emission are predicted in colliding wind binary scenarios depending on orbital parameters. The complex picture emerging from such simulations will be discussed and linked to proposed models for $\eta$ Car.

The 34th International Cosmic Ray Conference,

30 July- 6 August, 2015

The Hague, The Netherlands

\footnotetext{
*Speaker.
} 


\section{The case of colliding wind binaries in $\gamma$-ray astrophysics}

Detectable gamma-ray emission from colliding wind binaries has been predicted decades ago, and several systems were claimed to coincide with high-energy $\gamma$-ray sources observed by COS-B and EGRET, respectively. By now it has became clear that we fail to detect colliding wind binary systems as an object class in high-energy $\gamma$-ray astrophysics [1], with one truly remarkable exception, $\eta$ Carinae. Eta Carinae is commonly known as a binary system consisting of two massive stars (one being a member of the rare stellar class of luminous blue variables (LBV), the other being an $\mathrm{O}$ or WR star) with an orbital period of 5.54 yr. Both massive stars in the $\eta$ Car system produce powerful stellar winds. The respective mass-loss rates and terminal wind velocities are considered to be sufficiently high to form a wind-wind collision zone of shocked, hot gas, wherein particle acceleration in general [2] and subsequent $\gamma$-ray emission in particular can occur $[3,4,5]$. Conditions in the wind-wind collision zone depend on the orbital phase of the binary system, consequently one expects $\gamma$-ray emission that is principally modulated on orbital time scales. Additional complex dependencies, such as spectral cutoffs in the underlying particle spectrum due to excessive energy losses, anisotropy in the Inverse Compton process, or types of particle transport may add to the observational picture.

Since an unidentified $\gamma$-ray source was found in spatial coincidence of the enigmatic binary system $\eta$ Carinae [6,7], and distinct spectro-temporal characteristics of this source became evident [8], dedicated models explaining the emission in the $\gamma$-ray regime emerged $[9,10,11]$. Viable emission models are required to correspond to a plethora of observational features like the intensity and structure of the high-energy spectrum, energy-dependent orbital flux modulation and spectral cutoffs (Fig.1), an apparently asymmetric lightcurve over the first complete orbit [12], and ideally seek the connection to the rich phenomenology observed below the $\mathrm{MeV}$ range, e.g. most obviously regarding the presence of a nonthermal hard X-ray component [13, 14, 15].

With a full orbit of $\eta$ Carinae available in Fermi-LAT $\gamma$-ray data and better analysis performance owing to an improved instrument response function and background models, it is now possible to confront the observed phenomenology of $\eta$ Carinae at high energies with general as well as specific model predictions.

\section{Understanding the observed complex high-energy phenomenology}

The complex interplay between particle populations as function of energy, orbital phase and inclination etc. is nowadays best studied by hydrodynamical simulations in conjunction with particle transport models. To explain the observed phenomenology at high-energy $\gamma$-rays, two principally alternative scenarios able to account for the spectral variation observed for $\eta$ Carinae in the context of a colliding-wind binary interpretation were proposed. The spectral component emerging at periastron could be due to an additional (possibly hadronic) $\gamma$-ray emission component. Whereas the introduction of such component was driven to suit the purpose, such scenario appears nevertheless plausible in the light of systematic 3D-hydrodynamical studies that take into account the dynamics of the WCR and simultaneously solve a transport equation for shock-accelerated particles $[16,17]$. Although not yet applied to the specific parameters of the $\eta$ Carinae binary system, they demonstrate that considerable variations - even a transition from lepton to hadron dominated 

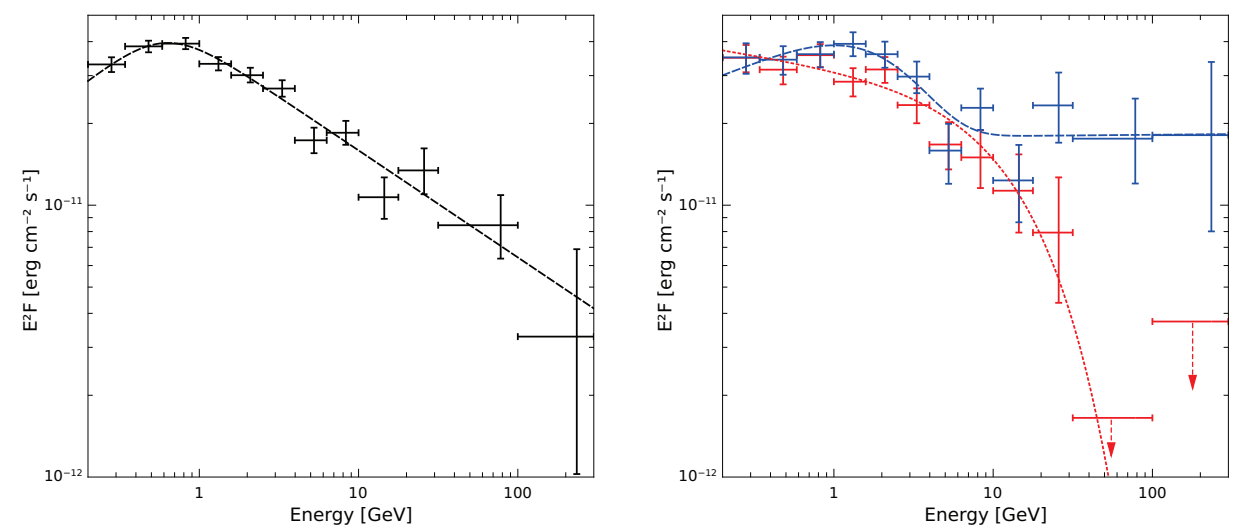

Figure 1: Spectral energy distribution of $\eta$ Carinae above $200 \mathrm{MeV}$. The line depicts the best fit spectral model to the Fermi-LAT data. On the left, the full orbit dataset is shown whereas on the right, 500 days centered on the periastron passage (blue) and the apastron passage (red) were selected.
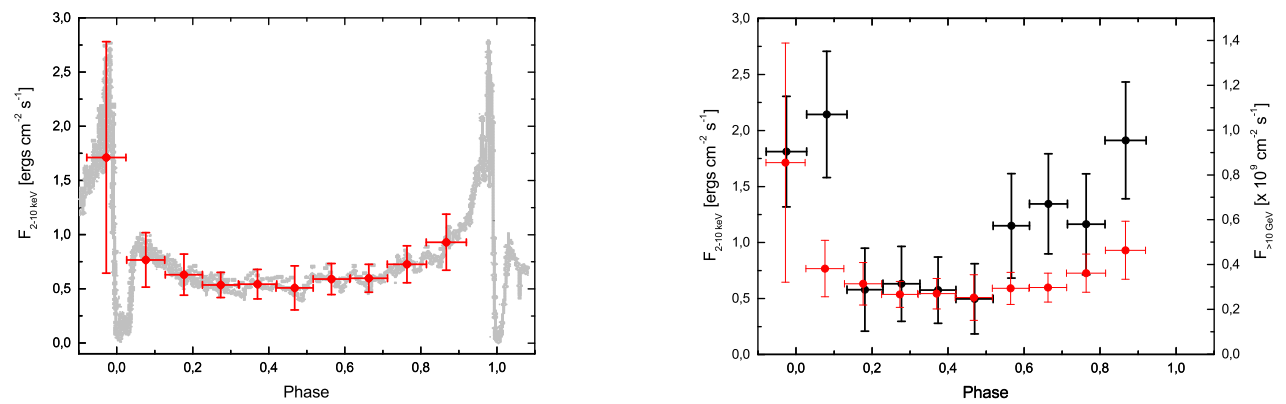

Figure 2: Full orbit lightcurve of $\eta$ Carinae. One the left, the 2003-2008 RXTE X-ray lightcurve is shown (black), subsequently integrated into the orbital intervals used in the Fermi-LAT analysis (red). On the right, the RXTE 2-10 keV X-ray lightcurve is shown (black), superimposed by the Fermi-LAT lightcurve above $50 \mathrm{GeV}$ in identical orbital phase bins.

$\gamma$-ray emission - may occur with changing stellar separation in a typical colliding-wind binary system. An alternative to a distinct second emission component emerging around periastron is still to be seen in a scenario of phase-modulated strong photon-photon absorption in the complex radiation fields in the vicinity of $\eta$ Carinae. The observed spectral variation can then be explained by the presence of the hot shocked gas in the WCR or by spatially extended X-ray emission components surrounding the system and the ensuing photon-photon absorption. Finally, once the RXTE X-ray lightcurve, integrated to the exact lightcurve bins as of [12], is compared with the Fermi GeVlightcurve, notable similarities as well as differences become apparent. Although models for the high-energy emission for $\eta$ Carinae have reached remarkable sophistication, one needs to conclude that none of proposed models are presently able to fully account for the observed phenomenology yet. 


\section{References}

[1] Werner, M.; Reimer, O.; Reimer, A.; Egberts, K. Fermi-LAT upper limits on gamma-ray emission from colliding wind binaries. Astronomy \& Astrophysics, Volume 555, pp. A102-A110 (2013)

[2] Eichler, D.; Usov, V. Particle acceleration and nonthermal radio emission in binaries of early-type stars. The Astrophysical Journal, Volume 402, no. 1, pp. 271-279 (1993)

[3] Benaglia, P.; Romero, G. E. Gamma-ray emission from Wolf-Rayet binaries. Astronomy \& Astrophysics, Volume 399, pp. 1121-1134(2003)

[4] Pittard, J. M.; Dougherty, S. M. Radio, X-ray, and gamma-ray emission models of the colliding-wind binary WR140. MNRAS, Volume 372, Issue 2, pp. 801-826(2006)

[5] Reimer, A.; Pohl, M.; Reimer, O. Nonthermal High-Energy Emission from Colliding Winds of Massive Stars. The Astrophysical Journal, Volume 644, Issue 2, pp. 1118-1144 (2006)

[6] Tavani, M. et al. Detection of Gamma-Ray Emission from the Eta-Carinae Region. The Astrophysical Journal Letters, Volume 698, Issue 2, pp. L142-L146 (2009)

[7] Abdo, A. A. et al. Fermi Large Area Telescope Observation of a Gamma-ray Source at the Position of Eta Carinae. The Astrophysical Journal, Volume 723, Issue 1, pp. 649-657 (2010)

[8] Reitberger, K.; Reimer, O.; Reimer, A.; Werner, M.; Egberts, K.; Takahashi, H. Gamma-ray follow-up studies on eta Carinae. Astronomy \& Astrophysics, Volume 544, pp. A98-A107 (2012)

[9] Bednarek, W.; Pabich, J. High-energy radiation from the massive binary system Eta Carinae. Astronomy \& Astrophysics, Volume 530, pp. A49-A59, 10 (2011)

[10] Farnier, C.; Walter, R.; Leyder, J.-C. eta Carinae: a very large hadron collider Astronomy \& Astrophysics, Volume 526, pp. A57-A64.(2011)

[11] Ohm, S.; Zabalza, V.; Hinton, J. A.; Parkin, E. R. On the origin of gamma-ray emission in eta Carina. MNRAS: Letters, Volume 449, Issue 1, p.L132-L136(2015)

[12] Reitberger, K.; Reimer, A.; Reimer, O.; Takahashi, H. The first full orbit of eta Carinae seen by Fermi. Astronomy \& Astrophysics, Volume 577, pp. A100-A105 (2015)

[13] Corcoran, M. F. X-Ray Monitoring of eta Carinae: Variations on a Theme. The Astronomical Journal, Volume 129, Issue 4, pp. 2018-2025. (2005)

[14] Leyder, J.-C.; Walter, R.; Rauw, G. Hard X-ray emission from eta Carinae. Astronomy and Astrophysics, Volume 477, Issue 3, 2008, pp. L29-L32 (2008)

[15] Hamaguchi, Kenji et al. Suzaku Monitoring of Hard X-Ray Emission from eta Carinae over a Single Binary Orbital Cycle. The Astrophysical Journal, Volume 795, Issue 2, pp. 119-130 (2014)

[16] Reitberger, K.; Kissmann, R.; Reimer, A.; Reimer, O.; Dubus, G. High-energy Particle Transport in Three-dimensional Hydrodynamic Models of Colliding-wind Binaries. The Astrophysical Journal, Volume 782, Issue 2, pp. 96-110(2014)

[17] Reitberger, K.; Kissmann, R.; Reimer, A.; Reimer, O.; Dubus, G. Simulating Three-dimensional Nonthermal High-energy Photon Emission in Colliding-wind Binaries. The Astrophysical Journal, Volume 789, Issue 1, pp. 87-106 (2014) 